\title{
Análisis de la Satisfacción y el Desempeño Laboral en los Funcionarios de la Municipalidad de Talcahuano
}

\section{ANALYSIS OF WORK SATISFACTION AND PERFORMANCE IN PUBLIC EMPLOYEES OF TALCAHUANO CITY HALL}

\author{
María Margarita Chiang Vega', Nadia Jacqueline San Martín Neira² \\ 1. Académica de recursos humanos de la Facultad de Ciencias Empresariales Universidad del Bio Bio. \\ 2. Ex-alumna del Magister en Dirección de Empresas de la Facultad de Ciencias Empresariales Universidad del Bio Bio.
}

\begin{abstract}
RESUMEN
Introducción: Dentro del área de recursos humanos para una institución pública es necesario medir las variables de desempeño y satisfacción laboral. Objetivo: medir cómo el desempeño laboral influye en la satisfacción laboral de los funcionarios. Materiales y métodos: se realizó un estudio no experimental, de diseño transversal, tipo descriptivo y correlacional. La información se obtuvo de 259 funcionarios de la municipalidad a quienes se aplicó un cuestionario estandarizado, personal, anónimo y voluntario. Resultados: La fiabilidad de las escalas varía desde meritorios a excelentes, con tamaño de Alfa de Cronbach entre 0,7 y 0,9. Los valores de desempeño laboral se distribuyen en 6 escalas, situando a los encuestados en una evaluación de "desempeño alta". Mientras que los valores de satisfacción laboral se distribuyen en 10 escalas, situando a los encuestados en un nivel de "laboralmente satisfechos", tanto para la muestra femenina como masculina. Conclusiones: El estudio encontró correlaciones estadísticamente significativas entre ambas variables, se destacan las correlaciones positivas entre las escalas de desempeño y satisfacción con la relación con el jefe, para el género femenino. Y las escalas de satisfacción con desempeño en la productividad, para el género femenino.
\end{abstract}

(Chiang M, San Martín N, 2015. Análisis de la Satisfacción y el Desempeño Laboral en los Funcionarios de la Municipalidad de Talcahuano. Cienc Trab. Sep-Dic; 17 [54]: 159-165).

Palabras claves: DESEMPEÑO LABORAL, SATISFACCIÓN LABORAL, MUNICIPALIDAD DE TALCAHUANO, FUNCIONARIOS PÚBLICOS.

\section{ABSTRACT}

Introduction: Within the human resources area for a public institution is compulsory to measure work performance and satisfaction variables. Objective: to measure how the job performance affects the employees' work satisfaction. Materials and Methods: The study followed a non-experimental descriptive and correlational design. The information was gathered from 259 public employees of the city hall who completed an anonymous and voluntary standardized questionnaire. Results: the reliability of scales vary from meritorious to excellent, with Cronbach's Alfa size between 0.7 and 0.9. The work performance values are distributed in 6 scales that place the responders in "high performance". While the job satisfaction values are distributed in 10 scales, and place the responders in "work satisfied" level. This applies equally to women and men. Conclusions: The study found statistically significant correlation between both variables, it can be highlighted the positive correlation between the performance scale and the satisfaction of the boss relation, for the female gender. And the satisfaction scale with the performance productivity, for the female gender.

Keywords: WORK PERFORMANCE, JOB SATISFACTION, TALCAHUANO CITY HALL, PUBLIC EMPLOYEES.

\section{INTRODUCCIÓN}

Ginebra comenta que los recursos humanos son el principal activo de la empresa; esto se puede deber a que todo tipo de organización está compuesta por personas y sin ellas no podrían existir. ${ }^{1}$ Es por ello que esta investigación se preocupa en conocer el desempeño y satisfacción laboral, siendo esta última una de las

Correspondencia / Correspondence:

Dra. Maria Margarita Chiang Vega

Universidad del Bio Bio

Académica de la Facultad de Ciencias Empresariales

Casilla 5-C, Concepción, Chile

Tel.: (41)3 111479

e-mail: mchiang@ubiobio.cl

Recibido: 28 de Octubre de 2015 / Aceptado: 10 de Noviembre de 2015

variables más estudiadas dentro de la sicología industrial y organizacional. Ello es reflejo de la importancia del tema tanto para los investigadores como para el público en general.

El motivo para elegir una municipalidad para esta investigación es porque durante las últimas dos décadas los municipios en Chile han comenzado a tener mayor importancia política, económica $\mathrm{y}$ social, donde actualmente deben asumir nuevos roles en un determinado territorio socialmente organizado, transformándose en un "facilitador del desarrollo local" (IULA/CELCADEL, 1993) generando estrategias que se traduzcan en acciones que beneficien a la comunidad en su conjunto. ${ }^{2}$

Frente a este desafío, la Municipalidad de Talcahuano ha sido reconocida por tener una excelente gestión municipal dentro de las comunas del país, siendo la mejor de toda la región del Bio Bio. ${ }^{2}$ Mediante el siguiente estudio, los directivos de la institución podrán saber los efectos que producen sus políticas, normas y procedimientos de la organización sobre el personal. Con la finalidad de mantener, suprimir, corregir o reforzar las políticas de la empresa, de acuerdo a los resultados obtenidos. 
Por lo anterior, el objetivo es medir cómo el desempeño laboral influye la satisfacción laboral de los funcionarios de la municipalidad de Talcahuano; este estudio se realiza con la finalidad de que el Municipio genere futuras políticas públicas regionales.

\section{Desempeño Laboral}

Milkovich y Boudrem definen desempeño como el grado en el cual el empleado cumple con los requisitos de trabajo. El autor Chiavenato expone que el desempeño es la eficacia del personal que trabaja dentro de las organizaciones, la cual es necesaria para la organización, funcionando el individuo con una gran labor y satisfacción laboral.

Chiang, Méndez y Sánchez comentan que existe una gran cantidad de criterios que podrían medirse al estimar el desempeño. Las capacidades, habilidades, necesidades y cualidades son características individuales que interactúan con la naturaleza del trabajo y de la organización para producir comportamientos, los cuales, a su vez, afectan los resultados. Como ejemplos de criterios para medir el desempeño de los empleados tenemos: cantidad de trabajo, calidad de trabajo, cooperación, responsabilidad, conocimiento del trabajo, asistencia, necesidad de supervisión, etc. El desempeño refleja principalmente los objetivos de la eficiencia, esto es, alcanzar las metas al tiempo que se utilizan los recursos eficientemente.

La Evaluación del Desempeño (ED), según Mondy y Noe, es un sistema formal de revisión y evaluación del desempeño laboral individual o de equipos. Mientras que Dolan, Valle, Jackson y Schuler comentan que es un procedimiento estructural y sistemático para medir, evaluar e influir sobre los atributos, comportamientos y resultados relacionados con el trabajo, así como el grado de absentismo, con el fin de descubrir en qué medida es productivo el empleado y si podrá mejorar su rendimiento futuro. Aunque la evaluación del desempeño de equipos es fundamental cuando éstos existen en una organización, el enfoque de la ED en la mayoría de las empresas se centra en el empleado individual. Sin importar el énfasis, un sistema de evaluación eficaz evalúa los logros e inicia planes de desarrollo, metas y objetivos.

En relación con la ED en la administración pública, Ramírez comenta que por lo general en las administraciones públicas el trabajo y el rendimiento son intrascendentes en lo que hace a la retribución y a la carrera de los funcionarios, siendo la evaluación, tal vez, el elemento de la Gerencia de Recursos Humanos que genera más pasiones, especialmente en el sector público.

\section{Satisfacción Laboral}

Desde la década de los 30 se registra un gran interés por la investigación en torno a la satisfacción en el trabajo, el cual alcanzó probablemente su punto máximo en los años 60. A partir de los años 70 se habla sobre los efectos en las condiciones de trabajo y los factores de la satisfacción; en los años 80 este tema empieza a situarse más con respecto a sus relaciones con la calidad de vida en el trabajo, en su impacto sobre la salud mental y en las relaciones entre éste y la familia, con una preocupación creciente por el desarrollo personal del individuo; finalmente, en los años 90, se observa una evolución hacia otro paradigma, el capital humano, destacando el constante desarrollo de ese capital mediante estrategias de formación.

Locke define la satisfacción laboral como un estado emocional positivo o placentero resultante de la percepción subjetiva de las experiencias laborales del sujeto o como respuesta afectiva de una persona a su propio trabajo.
Según Price y Muller identifican la satisfacción laboral con el grado en el que a los individuos les gusta su trabajo. Otros autores como Porter y Lawler expresan que es el resultado de la motivación con el desempeño del trabajo (grado en que las recompensas satisfacen las expectativas individuales) y de la forma en que el individuo percibe la relación entre esfuerzo y recompensa.

Finalmente, otra definición importante es la ofrecida por Davis y Newstrom, quienes definen la satisfacción en el trabajo como un conjunto de sentimientos favorables y desfavorables mediante los cuales los empleados perciben su trabajo. La satisfacción laboral es cambiante porque crecen y decrecen los sentimientos satisfactorios a medida que los motivos de logro se van cubriendo, por lo que las intensidades iniciales se complementan a través de las conductas realizadas.

Así, según Chiang, Martín y Núñez la satisfacción laboral es, básicamente, un concepto globalizador con el que se hace referencia a las actitudes de las personas hacia diversos aspectos de su trabajo. Por consiguiente, hablar de satisfacción laboral implica hablar de actitudes.

\section{MATERIALES Y MÉTODOS}

\section{Diseño de la investigación}

Esta investigación se clasifica como no experimental, ya que se realizó sin manipular las variables, observando los fenómenos para después analizarlos; además es de diseño transversal, ya que se realiza en un momento de tiempo determinado, de tipo descriptivo, debido a que se analizan y miden las variables, y correlacional, porque busca conocer la relación existente entre ambas variables.

\section{Descripción de la muestra}

El universo escogido para esta investigación corresponde a un total de 790 funcionarios de la Municipalidad de Talcahuano. Para definir el tamaño de la muestra se trabajó con un 95\% de confianza, se utilizó una varianza de un 0,5 y un 5\% de error, lo que define una muestra aleatoria de 259 funcionarios, cuya distribución por dirección, género y calidad jurídica se presentan en la Tabla 1.

Tabla 1.

Distribución de la muestra. Funcionarios de la casa matriz.

\begin{tabular}{|c|c|c|c|c|c|}
\hline \multirow[t]{2}{*}{ Dirección } & \multicolumn{2}{|c|}{$\begin{array}{l}\text { Planta /Contrata/ } \\
\text { Suplente }\end{array}$} & \multicolumn{2}{|c|}{ Honorarios } & \multirow[t]{2}{*}{ Total } \\
\hline & & Masc & Fem & Masc & \\
\hline Alcaldía & 0 & 4 & 0 & 0 & 4 \\
\hline Informática/Control & 1 & 7 & 2 & 0 & 10 \\
\hline DIDECO & 27 & 15 & 39 & 11 & 92 \\
\hline $\begin{array}{l}\text { Obras Municipales/ } \\
\text { Construcciones }\end{array}$ & 4 & 10 & 3 & 3 & 20 \\
\hline Adm. Municipal & 8 & 4 & 8 & 3 & 23 \\
\hline Aseo y Ornato & 1 & 4 & 0 & 0 & 5 \\
\hline SECPLAN & 1 & 4 & 12 & 11 & 28 \\
\hline Tránsito & 6 & 7 & 2 & 0 & 15 \\
\hline Adm. y Finanzas & 13 & 9 & 1 & 0 & 23 \\
\hline Asesoria Jurídica & 1 & 0 & 1 & 0 & 2 \\
\hline Cultura y Turismo & 2 & 2 & 0 & 0 & 4 \\
\hline Medio Ambiente & 2 & 2 & 4 & 1 & 9 \\
\hline Secretaria & 8 & 3 & 1 & 0 & 12 \\
\hline Juzgado policía & 9 & 1 & 2 & 0 & 12 \\
\hline TOTAL & 83 & 72 & 75 & $4^{29}$ & 259 \\
\hline
\end{tabular}




\section{Método de medición}

Los datos se recogieron aplicando un cuestionario de autorrespuesta anónima y confidencial, sin control de tiempo; se aplicó sin alterar el funcionamiento normal de las actividades cotidianas de manera voluntaria y anónima; esto permitió eliminar posibles influencias que pudieran inducir una respuesta determinada. Para tabular los datos se utilizó una planilla de cálculo en Excel, luego el paquete estadístico SPSS 20.

Parte I: Cuestionario de Desempeño Laboral. Para medir el desempeño de los funcionarios se adaptó un cuestionario desarrollado por Inostroza, con un total de 21 ítems, con una escala de 0 a 10, siendo 10 un desempeño elevado. En esta investigación los 21 ítems fueron factorizados en base al análisis del SPSS 20 y la teoría, lo que dio como resultado 6 factores o escalas: nivel de conocimiento respecto a la descripción del cargo, desempeño en la función, conocimiento de la organización, proactividad e innovación, relaciones interpersonales y productividad. (Ver Tabla 2). Parte II: Cuestionario de Satisfacción Laboral. Para medir la satisfacción de los funcionarios se utilizó un cuestionario desarrollado y validado por Chiang, Salazar, Huerta y Núñez, Chiang, Salazar, Martín y Núñez y Chiang, Gómez y Salazar, con un total de 44 ítems, los cuales se distribuyen en diez factores: satisfacción por el trabajo en general, Satisfacción por el ambiente físico, Satisfacción con la forma en cómo realiza el trabajo, Satisfacción

Tabla 2.

Fiabilidad de desempeño laboral.

$\begin{array}{lcc}\text { Factores } & N^{\circ} \text { de İtems } & \begin{array}{c}\text { Alpha de } \\ \text { Cronbach's }\end{array} \\ \text { 1. Nivel de conocimiento sobre la descripción del cargo } & 3 & 0,701 \\ \text { 2. Desempeño en la función } & 4 & 0,782 \\ \text { 3. Conocimiento de la institución } & 4 & 0,741 \\ \text { 4. Proactividad e innovación } & 5 & 0,836 \\ \text { 5. Relaciones interpersonales } & 2 & 0,880 \\ \text { 6. Productividad } & 3 & 0,764 \\ \quad \text { Cuestionario completo } & 21 & \end{array}$

Tabla 3.

Fiabilidad de satisfacción laboral.

\begin{tabular}{|c|c|c|c|c|c|c|}
\hline Factores & \multicolumn{2}{|c|}{$\begin{array}{l}\text { Chiang, Salazar, } \\
\text { Huerta y Núñez } \\
\text { (2008) }\end{array}$} & \multicolumn{2}{|c|}{$\begin{array}{l}\text { Chiang, Salazar, } \\
\text { Martín y Núñez } \\
\text { (2011) }\end{array}$} & \multicolumn{2}{|c|}{$\begin{array}{l}\text { Datos de la actual } \\
\text { investigación }\end{array}$} \\
\hline $\begin{array}{l}\text { Satisfacción por el } \\
\text { trabajo en general }\end{array}$ & 10 & 0,910 & 11 & 0,914 & 8 & 0,903 \\
\hline $\begin{array}{l}\text { Satisfacción con el } \\
\text { ambiente físico }\end{array}$ & 7 & 0,897 & 7 & 0,895 & 7 & 0,900 \\
\hline $\begin{array}{l}\text { Satisfacción con la } \\
\text { forma en cómo realiza } \\
\text { el trabajo }\end{array}$ & 6 & 0,868 & 6 & 0,877 & 6 & 0,878 \\
\hline $\begin{array}{l}\text { Satisfacción con la } \\
\text { oportunidad de desarrollo }\end{array}$ & 7 & 0,834 & 8 & 0,887 & 6 & 0,885 \\
\hline $\begin{array}{l}\text { Satisfacción con la } \\
\text { relación con jefe }\end{array}$ & 4 & 0,904 & 4 & 0,924 & 5 & 0,929 \\
\hline $\begin{array}{l}\text { Satisfacción con la } \\
\text { remuneración }\end{array}$ & 3 & 0,813 & 3 & 0,876 & 3 & 0,799 \\
\hline $\begin{array}{l}\text { Satisfacción con la } \\
\text { autonomía }\end{array}$ & 1 & * & 1 & * & 6 & 0,935 \\
\hline $\begin{array}{l}\text { Satisfacción con el } \\
\text { reconocimiento }\end{array}$ & 1 & * & 1 & * & 1 & * \\
\hline $\begin{array}{l}\text { Satisfacción por la } \\
\text { sección o área }\end{array}$ & - & - & - & - & 1 & * \\
\hline $\begin{array}{l}\text { Satisfacción por } \\
\text { la empresa }\end{array}$ & - & - & - & - & 1 & * \\
\hline Cuestionario completo & 39 & 41 & 46 & & & \\
\hline
\end{tabular}

con la oportunidad de desarrollo, Satisfacción con la relación con el jefe, Satisfacción con la remuneración, Satisfacción con la autonomía, Satisfacción con el reconocimiento, Satisfacción por la sección o área y Satisfacción por la empresa. Los funcionarios responden utilizando un formato de respuesta tipo Likert de cinco puntos, siendo 5 Satisfacción Total. (Ver Tabla 3).

\section{RESULTADOS}

Análisis de fiabilidad (coeficiente de consistencia interna o indicador de unidimensionalidad)

En las tablas 2 y 3 se observan las estimaciones de la consistencia interna de las escalas de desempeño y satisfacción laboral. La fiabilidad de las escalas es variable y en todos los casos es adecuada; estos resultados están entre 0,7 y 0,9 , es decir, las escalas son consistentes. La Tabla 3 (en las columnas, 2, 3, 4 y 5) muestra resultados históricos a modo de conocer la fiabilidad de las escalas en otras muestras.

\section{Análisis descriptivo}

Resultados sobre la información general de la muestra

Del total de los funcionarios, el 61\% (158/259) son mujeres y un $39 \%(101 / 259)$ son hombres, con una edad promedio de 43 años y 13 años de antigüedad en la municipalidad. En relación a la calidad jurídica, un 38\% de los funcionarios pertenece a planta, siendo en su mayoría hombres con un 47,5\%, seguido de un 37\% de los funcionarios que pertenecen a honorarios, siendo en su mayoría mujeres con un 43,7\%. En cuanto al estamento, un 37\% pertenece a profesionales, seguido por un 32\% de administrativos para ambos géneros.

Mientras que en educación, los funcionarios de ambos géneros poseen estudios universitarios completos, representando un 30\%.

Resultados de desempeño laboral

Respecto a los promedios del desempeño laboral (ver Tabla 4) se miden seis factores, la escala de medición de cada item es de 0 a 10, siendo 10 la evaluación más alta; cada escala de desempeño laboral posee diferentes cantidades de ítems. En general, los niveles de percepción del desempeño laboral en los funcionarios de la muestra están sobre la media (6, en una escala que va del 0 al 10). Es decir, no hay resultados que indiquen niveles de desempeño bajo. Respecto a los promedios en los departamentos de la institución, hay que destacar que ambos géneros y en todas las direcciones (6/6) su evaluación se encuentra sobre la media, es decir, poseen un desempeño alto $(6,57-8,20$, con un máximo de 10$)$.

Por otra parte, un análisis por escala arroja que tanto en la muestra femenina como masculina, de los funcionarios, sus valores más altos se encuentran en los factores "Desempeño sobre las relaciones interpersonales" y "Desempeño sobre la productividad" con puntuación de 8,13 y 8,0 respectivamente. En tanto que el "conocimiento de la organización" alcanza el valor más bajo para ambos géneros, correspondiendo a 6,81, siendo los ítem "conocimiento sobre el manual de procedimientos y reglamento interno de higiene y seguridad" los más bajos.

De acuerdo a los promedios obtenidos sobre el Desempeño Laboral en ambos géneros, las mujeres son más exigentes al momento de evaluar su desempeño laboral (2 promedios sobre 6) 
Tabla 4. Promedios desempeño laboral.

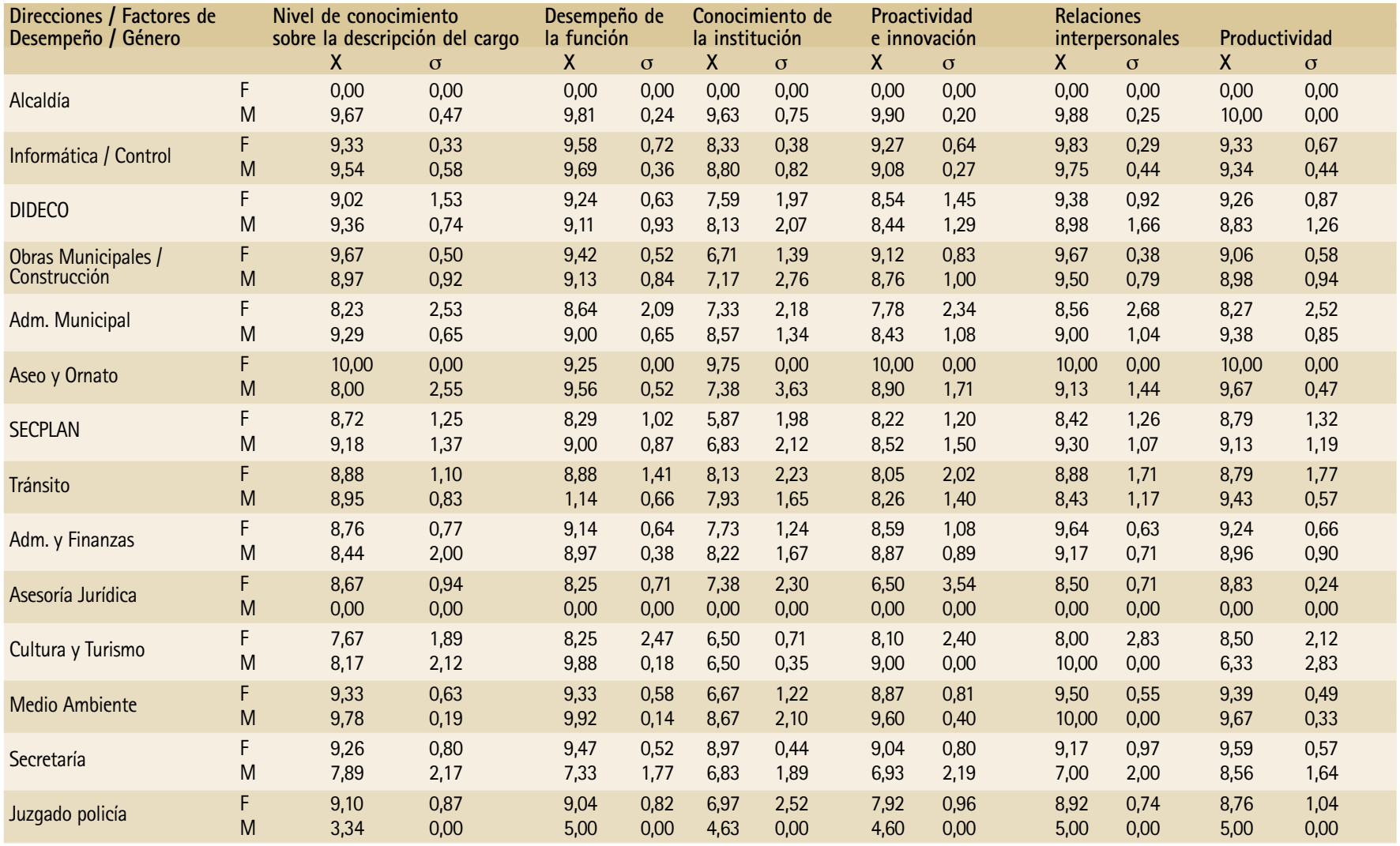

Tabla 4. Promedios satisfación laboral.

\begin{tabular}{|c|c|c|c|c|c|c|c|c|c|c|c|c|c|c|c|c|c|c|c|c|c|}
\hline \multirow{2}{*}{$\begin{array}{l}\text { Direcciones* } \\
\text { Factores / } \\
\text { Género }\end{array}$} & & \multicolumn{2}{|c|}{$\begin{array}{l}\text { Trabajo } \\
\text { en general }\end{array}$} & \multicolumn{2}{|c|}{$\begin{array}{l}\text { Ambiente } \\
\text { Físico }\end{array}$} & \multicolumn{2}{|c|}{$\begin{array}{l}\text { Forma en cómo } \\
\text { realiza el trabajo }\end{array}$} & \multicolumn{2}{|c|}{$\begin{array}{l}\text { Oportunidad } \\
\text { de desarrollo }\end{array}$} & \multicolumn{2}{|c|}{$\begin{array}{l}\text { Relación } \\
\text { con el jefe }\end{array}$} & \multicolumn{2}{|c|}{ Remuneración } & \multicolumn{2}{|c|}{ Autonomía } & \multicolumn{2}{|c|}{ Reconocimiento } & \multicolumn{2}{|c|}{$\begin{array}{l}\text { Sección } \\
\text { o área }\end{array}$} & \multicolumn{2}{|c|}{$\begin{array}{l}\text { Por la } \\
\text { Empresa }\end{array}$} \\
\hline & & $\vec{x}$ & $\sigma$ & $x$ & $\sigma$ & $\mathrm{X}$ & $\sigma$ & $x$ & $\sigma$ & $x$ & $\sigma$ & $\mathrm{x}$ & $\sigma$ & $\mathrm{x}$ & $\sigma$ & $\mathrm{X}$ & $\sigma$ & $x$ & $\sigma$ & $x$ & $\sigma$ \\
\hline \multirow[t]{2}{*}{1} & $\mathrm{~F}$ & 0,00 & 0,00 & 0,00 & 0,00 & 0,00 & 0,00 & 0,00 & 0,00 & 0,00 & 0,00 & 0,00 & 0,00 & 0,00 & 0,00 & 0,00 & 0,00 & 0,00 & 0,00 & 0,00 & 0,00 \\
\hline & M & 4,59 & 0,28 & 4,57 & 0,51 & 4,71 & & 4,58 & 0,35 & 4,75 & 0,38 & 4,67 & 0,38 & 4,67 & 0,36 & 5,00 & 0,00 & 4,50 & 0,58 & 3,75 & 1,89 \\
\hline \multirow[t]{2}{*}{2} & $\mathrm{~F}$ & 4,67 & 0,31 & 4,38 & 0,54 & 4,67 & 0,29 & 4,50 & 0,44 & 4,93 & 0,12 & 4,00 & 0,88 & 4,83 & 0,17 & 4,67 & 0,58 & 5,00 & 0,00 & 4,33 & 0,58 \\
\hline & M & 4,52 & 0,35 & 4,03 & 0,71 & 4,46 & 0,26 & 3,74 & 0,46 & 4,60 & 0,20 & 3,64 & 0,67 & 4,32 & 0,37 & 4,60 & 0,40 & 4,70 & 0,25 & 3,95 & 0,81 \\
\hline \multirow[t]{2}{*}{3} & $\mathrm{~F}$ & 3,54 & 0,86 & 3,37 & 1,05 & 78 & 0,87 & 3,06 & 1,05 & 3,67 & 1,03 & 3,23 & 1,14 & 3,38 & 1,08 & 3,18 & & 3,32 & 1,12 & 3,40 & 1,10 \\
\hline & M & 3,63 & 0,87 & 3,96 & 0,79 & 3,96 & 0,74 & 3,39 & 0,88 & 3,81 & 0,88 & 3, & 0,84 & 3,67 & 0,89 & 3,62 & 1,13 & 3,81 & 0,98 & 3,88 & 0,95 \\
\hline 4 & M & 08 & 0,64 & 3,47 & 0,80 & & 0,52 &, 50 & 1,08 & 4,36 & 0,69 & & 0,83 & 4,01 & 0,72 & 4,16 & & 7,25 & 0,65 & 3,95 & 1,04 \\
\hline \multirow[t]{2}{*}{5} & $\mathrm{~F}$ & 3,82 & 0,79 & 3,98 & 0,72 & 4,23 & 0,66 & 3,42 & 0,95 & 3,94 & 0,93 & 3,67 & 1,14 & 4,00 & 0,81 & 3,81 & 0,9 & 3,94 & 0,93 & 3,88 & 1,15 \\
\hline & M & 4,07 & 0,91 & 4,08 & 0,86 & & 0,83 & 3,69 & 1,07 & 4,03 & 1,26 & & 0,9 & 95 & 1,43 & 4,14 & & ;71 & 1,38 & 4,14 & 1,07 \\
\hline \multirow[t]{2}{*}{6} & $\mathrm{~F}$ & 4,38 & 0,00 & 3,14 & 0,00 & & $0, C$ & & 0,00 & 5,00 & 0,00 & & 0,0 & 4,6 & 0,0 & 5,00 & 0,0 & 5,00 & 0,00 & 5,00 & 0,00 \\
\hline & M & 3,81 & 0,75 & 4,00 & 0,69 & & & & 0,62 & 4,3 & 1,0 & & 0,3 & & 0 , & 4,25 & & 4,00 & 0,82 & 3,50 & 0,58 \\
\hline 7 & $\mathrm{~F}$ & 3,99 & 0,56 & 4,04 & 0,74 & & 0,70 & 3,53 & 0,94 & 4,23 & 0,60 & 3,44 & 0,88 & 4,04 & 0,70 & 3,85 & 0,95 & 3,92 & 0,76 & 3,85 & 0,90 \\
\hline 9 & M & 3,90 & 0,59 & 3,65 & 0,60 & 391 & 0,76 & 3,63 & 0,87 & 3,96 & 0,65 & 3,63 & 0,72 & 3,89 & 0,68 & 3,78 & 0,83 & 3,78 & 0,67 & 3,89 & 1,27 \\
\hline \multirow[t]{2}{*}{10} & $\mathrm{~F}$ & 4,69 & 0,09 & 4,50 & 0,51 & & 0,00 & 4,58 & 0,12 & 5,00 & 0,00 & 4,8 & 0,24 & 4,67 & 0,00 & 5,00 & 0,0 & 5,00 & 0,00 & 4,50 & 0,71 \\
\hline & M & & 0,00 & 0,00 & 0,00 & & & 0,00 & 0,00 & 0,00 & 0,00 & & 0,0 & 0,0 & 0,0 & 0,00 & 0,0 & 0,00 & 0,00 & 0,00 & 0,00 \\
\hline \multirow[t]{2}{*}{11} & $\mathrm{~F}$ & & 1,24 & & 0,71 & & & & & 3,8 & & & & & & 4,00 & & 4,00 & 1,41 & 4,50 & 0,71 \\
\hline & M & 3,94 & 0,44 & 4,00 & 0,20 & 4,0 & 1,3 & 3,42 & 0,82 & 4,60 & 0,28 & 3,8 & 1,1 & 4,67 & 0,47 & 4,50 & 0,71 & 4,50 & 0,71 & 3,00 & 2,83 \\
\hline 12 & $\mathrm{~F}$ & 3,81 & 0,58 & 3,29 & 0,37 & & 0,2 & 3,22 & 0,50 & 3,90 & 0,52 & 3,00 & 0,5 & 3,97 & 0,37 & 3,67 & 0,52 & 4,00 & 0,63 & 3,83 & 0,41 \\
\hline & M & & 0,38 & 3,67 & 0,84 & & 0,69 & 2,83 & 0,33 & 4,07 & 0,12 & 2,78 & 0,19 & 4,06 & 0,48 & 3,67 & 0,58 & 4,00 & 1,00 & 3,33 & 0,58 \\
\hline 13 & $\mathrm{~F}$ & & 0,29 & 3,40 & 0,89 & & 0,29 & 3,57 & 0,52 & 4,53 & 0,36 & 3,78 & 0,83 & 4,39 & 0,46 & 4,11 & 0,33 & 4,44 & 0,53 & 4,00 & 0,50 \\
\hline & M & & 0,38 & 4,29 & 0,49 & & & & 0,75 & 4,60 & & & 0,5 & & 0,54 & 4,67 & & 4,67 & 0,58 & 4,33 & 0,58 \\
\hline 14 & $\mathrm{~F}$ & 3,45 & 0,69 & 2,99 & 0,95 & 3,62 & 0,87 & 2,76 & 0,57 & 3,81 & 0,77 & 2,91 & 0,91 & 3,10 & 0,76 & 3,72 & 1,14 & 3,39 & 1,10 & 3,34 & 1,16 \\
\hline & M & 3,37 & 0,00 & 3,00 & 0,00 & 3,33 & 0,00 & 2,16 & 0,00 & 3,60 & 0,00 & 2,33 & 0,00 & 2,00 & 0,00 & 4,00 & 0,00 & 3,00 & 0,00 & 1,00 & 0,00 \\
\hline
\end{tabular}

Nota (Direcciones*): La enumeración de las direcciones corresponde a la misma enumeración que existe en la Tabla № 3 . 
a diferencia del género masculino (4 promedios sobre 6), que presentó una autoevaluación mayor sobre su desempeño. (Hay que destacar que cada funcionario evaluó su desempeño dentro de sus funciones laborales, por lo que existe un sesgo).

\section{Resultados de satisfacción laboral}

Respecto a los promedios de la satisfacción laboral (ver Tabla 5), la escala de medición de cada ítem es de 1 a 5 , significando 5 "totalmente satisfecho"; cada escala de satisfacción laboral posee diferentes cantidades de ítems. En general, los niveles de percepción de la satisfacción laboral en los funcionarios de la muestra están en la media (3, en una escala que va del 1 al 5). Es decir, no hay resultados que indiquen niveles de satisfacción bajo.

Respecto a los promedios en los departamentos de la institución, hay que destacar que ambos géneros y en todas las direcciones (10/10) su evaluación se encuentra sobre la media $(3,03-3,78$, de un máximo de 5), es decir, los funcionarios se encuentran satisfechos laboralmente.

Por otra parte, un análisis por escala arroja que tanto en la muestra femenina como masculina, de los funcionarios, sus valores más altos se encuentran en los factores "Satisfacción con la relación con el jefe" con puntuación de 3,76, respectivamente. En tanto que, el "Satisfacción por la remuneración" alcanza el valor más bajo para el género femenino, correspondiendo a 3,06 y el "Satisfacción por las oportunidades de desarrollo" alcanza el valor más bajo para el género masculino, correspondiendo a 3,11. De acuerdo a los promedios obtenidos sobre la Satisfacción Laboral de ambos géneros, las mujeres son más detallista y exigentes al momento de evaluar su satisfacción laboral por lo que su evaluación es menor (4 promedios sobre 10), a diferencia del género masculino que presentó una satisfacción mucho mayor (6 promedios sobre 10 ).

\section{Análisis de correlaciones: Relación entre desempeño laboral y los factores de satisfacción laboral}

En las tablas 6 y 7 se observan las correlaciones de ambos géneros, entre las dimensiones de desempeño laboral y satisfacción laboral. Respecto a la relación existente entre las dos variables estudiadas, los resultados muestran correlaciones positivas y estadísticamente significativas, en niveles moderados y altos para el género femenino (ver Tabla 6), donde las variables "proactividad e innovación" poseen una correlación estadísticamente significativa positiva con "Satisfacción con la relación con el jefe" y "Satisfacción con el reconocimiento", es decir, a medida que en las funcionarias aumenten su proactividad e innovación en su trabajo, aumenta la satisfacción con la relación que tienen con sus superiores y con el reconocimiento. Por otro lado, existe una correlación positiva y estadísticamente positiva entre todos los factores de desempeño laboral y la "satisfacción con la relación con el jefe"; esto podría significar que cuando se aumenta el desempeño dentro de las funcionarias, existe un aumento de la satisfacción respecto a la relación que llevan con sus superiores en la institución. Para el género masculino (ver Tabla 7), los resultados muestran correlaciones positivas y estadísticamente significativas, aunque en niveles moderados y aceptables, donde las variables "proactividad e innovación" poseen una correlación estadísticamente significativa positiva alta con "Satisfacción por la empresa", es decir, a medida que los funcionarios aumentan su desempeño en actividades relacionadas con la proactividad e innovación aumentará su satisfacción por trabajar en la institu- ción. Por otro lado, existe una correlación positiva y estadísticamente positiva entre productividad y la mayoría de los factores de satisfacción laboral; esto podría significar que los funcionarios, al aumentar su productividad, aumentan su satisfacción respecto al ambiente físico, la forma en que realizan su trabajo, oportunidades de desarrollo, relación con sus superiores, autonomía y satisfacción por trabajar en la municipalidad.

\section{DISCUSION}

\section{Conclusiones}

El objetivo de esta investigación es analizar la relación existente entre el desempeño laboral y la satisfacción laboral en los funcionarios de la municipalidad de Talcahuano; de los resultados obtenidos se puede concluir lo siguiente:

Respecto a los niveles de percepción obtenidos sobre el desempeño laboral en los funcionarios de la muestra están sobre la media (6, en una escala que va del 0 al 10), es decir, no existen niveles bajos de desempeño. Ambos géneros coinciden en que se preocupan por mantener relaciones interpersonales que alimenten un grato ambiente laboral junto a sus compañeros de trabajo y en tener menor conocimiento respecto al reglamento de higiene $\mathrm{y}$ seguridad, y manual de procedimientos.

Respecto a los niveles de percepción obtenidos sobre la satisfacción laboral en general, los valores están entre 3,2 y 3,6. Estos indicadores muestran que los niveles en los funcionarios están sobre la media (3, en una escala que va del 1 al 5), es decir, existe satisfacción. Cuando se comparan ambos géneros coinciden en tener una mayor satisfacción en la relación que mantienen con sus superiores, el reconocimiento que se les entrega, y por trabajar dentro de ese departamento, dirección o institución. Por otro lado, presentan una menor satisfacción respecto a las políticas preventivas que aplica la institución.

En la relación existente entre las dos variables estudiadas para esta muestra, los resultados muestran correlaciones estadísticamente significativas, en general desde niveles moderados a altos, donde se aprecia en el género femenino una mayor cantidad de correlaciones moderadas y altas con un 35\% del total a diferencia del género masculino con un $27 \%$ del total.

Para el género femenino se aprecia que las correlaciones que más destacan son las relaciones entre la satisfacción con el jefe y las escalas de desempeño laboral (todas). Además, se refleja que si aumenta el desempeño, principalmente la proactividad e innovación en las funcionarias de la municipalidad, aumentará la satisfacción por obtener reconocimiento y satisfacción por las relaciones que se establezcan son sus superiores (ver Tabla 6). Lo anterior tiene relación con la teoría de los dos factores de Herzberg (1959), específicamente con los factores intrínsecos o motivadores (surgen de los esfuerzos del trabajador y afectan la satisfacción), es decir, para que aumenten su desempeño las mujeres necesitan sentirse estimuladas por sus propios esfuerzos como: reconocimiento, autonomía, crecimiento, logros, proactividad, oportunidades de innovación e identidad en el trabajo.

Por otro lado, en el género masculino se destacan las correlaciones que indican que si aumenta el desempeño en la productividad de los funcionarios y en el desempeño en el cumplimiento de los objetivos del cargo, aumentará la satisfacción por el ambiente físico, la satisfacción con la relación con sus superiores y la satisfacción por trabajar en la municipalidad (ver Tabla 7). Lo 
Tabla 6. Correlación de satisfacción laboral con desempeño laboral (género femenino).

\begin{tabular}{|c|c|c|c|c|c|c|}
\hline ESCALAS & $\begin{array}{l}\text { Nivel de conocimiento sobre } \\
\text { la descripción del cargo }\end{array}$ & $\begin{array}{l}\text { Desempeño en } \\
\text { la función }\end{array}$ & $\begin{array}{l}\text { DESEMPEÑO LAB } \\
\text { Conocimiento } \\
\text { de la institución }\end{array}$ & $\begin{array}{l}\text { 30RAL } \\
\text { Proactividad } \\
\text { e innovación }\end{array}$ & $\begin{array}{l}\text { Relaciones } \\
\text { interpersonales }\end{array}$ & Productividad \\
\hline Satisfacción por el trabajo en general & $\begin{array}{c}0,260 \\
(0,314)\end{array}$ & $\begin{array}{c}0,466 \\
(0,060)\end{array}$ & $\begin{array}{c}0,742^{* *} \\
(0,001)\end{array}$ & $\begin{array}{c}0,416 \\
(0,097)\end{array}$ & $\begin{array}{c}0,328 \\
(0,198)\end{array}$ & $\begin{array}{c}0,266 \\
(0,301)\end{array}$ \\
\hline Satisfacción con el ambiente fisico & $\begin{array}{l}0,360 \\
(0,156)\end{array}$ & $\begin{array}{c}0,369 \\
(0,145)\end{array}$ & $\begin{array}{c}0,384 \\
(0,128)\end{array}$ & $\begin{array}{l}0,517^{*} \\
(0,034)\end{array}$ & $\begin{array}{l}0,493^{*} \\
(0,044)\end{array}$ & $\begin{array}{c}0,114 \\
(0,662)\end{array}$ \\
\hline Satisfacción con la forma en cómo realiza el trabajo & $\begin{array}{c}0,291 \\
(0,257)\end{array}$ & $\begin{array}{l}0,541^{*} \\
(0,025)\end{array}$ & $\begin{array}{l}0,773^{* *} \\
(0,000)\end{array}$ & $\begin{array}{c}0,351 \\
(0,167)\end{array}$ & $\begin{array}{c}0,417 \\
(0,096)\end{array}$ & $\begin{array}{c}0,225 \\
(0,386)\end{array}$ \\
\hline Satisfacción con la relación con jefe & $\begin{array}{l}0,552^{*} \\
(0,022)\end{array}$ & $\begin{array}{l}0,665^{* *} \\
(0,004)\end{array}$ & $\begin{array}{l}0,562^{*} \\
(0,019)\end{array}$ & $\begin{array}{l}0,796^{* *} \\
(0,000)\end{array}$ & $\begin{array}{l}0,742^{* *} \\
(0,001)\end{array}$ & $\begin{array}{l}0,496^{*} \\
(0,043)\end{array}$ \\
\hline Satisfacción con la remuneración & $\begin{array}{c}0,159 \\
(0,543)\end{array}$ & $\begin{array}{c}0,004 \\
(0,989)\end{array}$ & $\begin{array}{c}0,319 \\
(0,212)\end{array}$ & $\begin{array}{l}0,095 \\
(0,718)\end{array}$ & $\begin{array}{c}0,203 \\
(0,435)\end{array}$ & $\begin{array}{c}0,324 \\
(0,204)\end{array}$ \\
\hline Satisfacción con la autonomía & $\begin{array}{l}0,364 \\
(0,151)\end{array}$ & $\begin{array}{l}0,511^{*} \\
(0,036)\end{array}$ & $\begin{array}{l}0,679^{* *} \\
(0,003)\end{array}$ & $\begin{array}{c}0,453 \\
(0,068)\end{array}$ & $\begin{array}{c}0,306 \\
(0,232)\end{array}$ & $\begin{array}{c}0,301 \\
(0,240)\end{array}$ \\
\hline Satisfacción por la empresa & $\begin{array}{l}0,556^{*} \\
(0,021)\end{array}$ & $\begin{array}{c}0,261 \\
(0,312)\end{array}$ & $\begin{array}{l}0,237 \\
(0,360)\end{array}$ & $\begin{array}{l}0,540^{*} \\
(0,025)\end{array}$ & $\begin{array}{c}0,478 \\
(0,052)\end{array}$ & $\begin{array}{l}0,218 \\
(0,402)\end{array}$ \\
\hline
\end{tabular}

*. La correlación es significativa al nivel 0,01 (bilateral). *. La correlación es significativa al nivel 0,05 (bilateral).

Tabla 7. Correlación de satisfacción laboral con desempeño laboral (género masculino).

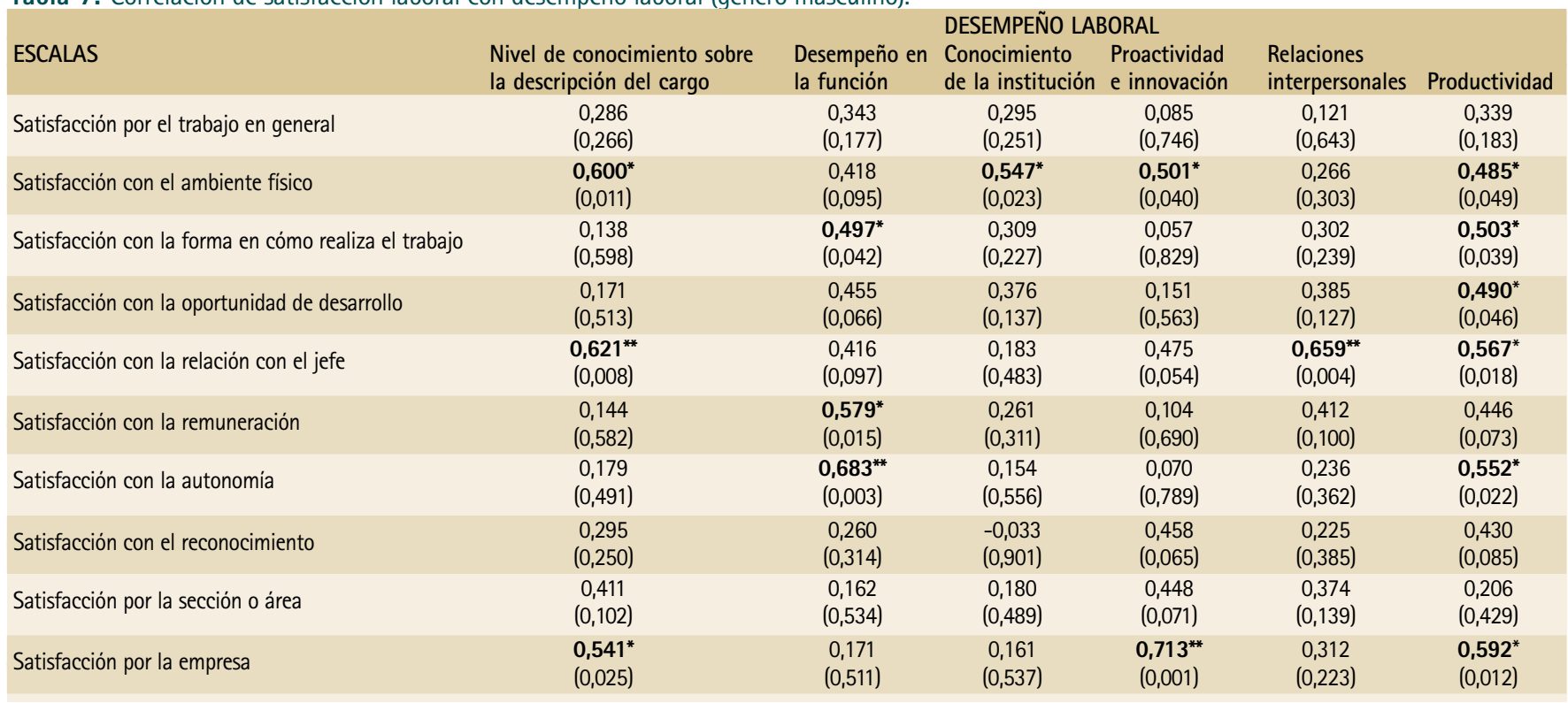

*. La correlación es significativa al nivel 0,01 (bilateral). *. La correlación es significativa al nivel 0,05 (bilateral).

anterior también posee relación con la teoría de los dos factores de Herzberg (1959), específicamente con los factores extrínsecos o higiénicos (prosiguen de los esfuerzos del trabajador, cuya ausencia ocasiona insatisfacción), es decir, que si los funcionarios de la municipalidad se preocupan de desempeñar su trabajo como corresponde, a futuro recibirán los beneficios esperados como: status, salario, mejorar su relación con sus superiores, mejores condiciones laborales y satisfacción por la institución.

Los hallazgos presentes en este trabajo muestran que el desempeño laboral en las mujeres es afectado por las actividades que logren estimularlas y causar una satisfacción laboral (Factores intrínsecos), es decir, las funcionarias necesitan el crecimiento individual, encontrar una identidad, tener reconocimiento profesional, crear relaciones interpersonales con sus compañeros de trabajo y superiores, y la necesidad de autorrealización dentro de la municipalidad. La ausencia de esto, no provoca insatisfacción, sino un estado de no satisfacción. A diferencia de los hombres, su desempeño laboral es afectado por las condiciones, perfil del cargo y decisiones que determine la municipalidad, es decir, la remuneración, beneficios, políticas de la empresa, supervisión, ambiente físico, etc. (Factores extrínsecos). La ausencia de esto podría ocasionar insatisfacción, pero carecen de capacidad para aumentar o mejorar la satisfacción.

Desde el punto de vista académico, la investigación ha permitido: (1) realizar una integración de la literatura de las variables en estudio, las cuales han permitido abordar la investigación en profundidad; (2) conocer y analizar los conceptos, para luego adaptar las herramientas de medición para las variables; (3) aportar desde un punto 
de vista teórico y empírico en la medición del desempeño y satisfacción laboral, con la finalidad de conocer su relación.

Esta investigación permite la toma de decisiones dentro de la municipalidad para generar futuras políticas públicas internas, a partir de lo que perciben los funcionarios(as) al momento de realizar su trabajo. En este sentido, se ofrece un marco de referencia en el que se plantean un conjunto de variables que se deberán analizar, y sugerencias para poder aumentar el desempeño y satisfacción de los funcionarios. Por ejemplo, se ofrece la posibilidad del análisis riguroso a los funcionarios de la institución, en el ámbito de crear indicadores que permitan medir el desempeño de acuerdo a diferentes criterios de evaluación. ${ }^{4}$ También es recomendable replicar esta investigación en otras instituciones públicas en general.

\section{Agradecimientos}

Los investigadores agradecen a la municipalidad de Talcahuano por autorizarnos a utilizar su nombre dentro del estudio y a Paola Patricia Merino Herrera por la traducción al inglés del resumen.

Proyecto de investigación 1310183/R Universidad del Bio Bio, Concepción, Chile.

\section{REFERENCES}

1. Proyecto SACDEL-Federación de Municipios de Canadá. Seminario Internacional: EI Municipio como Promotor del Desarrollo Económico Local;16-19 feb; San José, Costa Rica [en línea]. Quito: IULA/CELCADEL;1993 [consultado 15 jul 2015]. Cuaderno de Desarrollo Local; 13. Disponible en: http://www.flacmabibliotecamunicipalvirtual.com/images/flacmabiblioteca/Libros/Edicion\%2013\%20El\%20Municipio\%20Como\%20Promotor\%20del\%20Desarrollo\%20Economico\%20Local.pdf

2. Milkovich G, Boudreau J. Dirección y Administración de Recursos Humanos. Un enfoque de estrategia.6a ed. México,DF: Addison-Wesley lberoamericana; 1994.

3. Chiavenato I. Gestión de talento humano. Bogotá: McGraw-Hill; 2002.

4. Mondy R, Noe R. Administración de Recursos Humanos. 9a ed. México: Pearson Educación; 2005.

5. Chinchilla, K. Un sistema de evaluación del desempeño: aspectos a considerar en su diseño. 5campus.org-CiberConta.com [en línea]. Zaragoza: Universidad de Zaragoza; 2000 [consultado 2 jul 2015]. Disponible en: http://www.5campus. org/leccion/sievade. Cita a: Dolan S, Valle R, Jackson S y Schuler R. La gestión de los recursos humanos. 3a ed. España: McGraw-Hill; 2007.

6. Ramirez C. Gestión estratégica de recursos humanos y evaluación del desempeño en la administración pública chilena. Rev Gobierno y administración del Estado [en línea] 2000 [consultado 11 jul 2015]; 4(88):57-81. Disponible en: http://es.slideshare.net/cheramig/crg-evaluacin-del-desempeo-y-gestin-estratgica-de-rrhh

7. Locke EA. The nature and causes of job satisfaction. En: Dunnette M, editor. Handbook of industrial and organizational psychology. Chicago: Rand McNally; 1976. p.1297-1349.
8. Price JL, Mueller CW. Handbook of organizational measurement. Marshfield : Pitman; 1986.

9. Porter LM, Lawler EE. Actitud empresarial y desempeño. New York: McGrawHill; 1991.

10. Davis K, Newstrom W. El comportamiento humano en el trabajo; Comportamiento organizacional. México: McGraw- Hill; 2007.

11. Inostroza K. Estudio de la relación entre el clima organizacional, desempeño y rotación laboral en el SENAME de Concepción [Tesis]. Concepción: Universidad del Bio Bio; 2012.

12. Chiang $M$, Salazar $M$, Huerta $P$, Núñez $A$. Clima organizacional y satisfacción laboral en organizaciones del sector estatal (instituciones públicas). Desarrollo, adaptación y validación de instrumentos. Universum Talca [en línea] 2008 [consultado 02 jul 2015]; 23(2):66-85. Disponible en: http://www.scielo.cl/ scielo.php?pid=S0718-23762008000200004\&script=sci_arttext

13. Chiang $M$, Salazar $M$, Martín MJ, Núñez A. Clima organizacional y satisfacción laboral, una comparación entre hospitales públicos de alta y baja complejidad. Salud Trab [en línea] 2011 [consultado 09 jul 2015]; 19(1):5-16. Disponible en: http://dialnet.unirioja.es/servlet/articulo?codigo $=3707988$

14. Chiang M, Gómez N, Salazar C. Satisfacción laboral y estilos de liderazgo en instituciones públicas y privadas de educación en Chile. Cuad Adm [en línea] 2014 [consultado 23 jul 2015]; 30(52):65-74. Disponible en: http://www.scielo. org.co/scielo.php?script=sci_arttext\&pid=S0120-46452014000200007 \&lng=es\&tlng= 